\title{
Could electron-positron annihilation lines in the Galactic center result from pulsar winds?
}

\author{
W. Wang ${ }^{1,3}$, C. S. J. Pun ${ }^{2}$, and K. S. Cheng ${ }^{2}$ \\ 1 Max-Planck-Institut für extraterrestrische Physik, Postfach 1312, 85741 Garching, Germany \\ e-mail: wwang@mpe.mpg.de \\ 2 Department of Physics, University of Hong Kong, Pokfulam Road, Hong Kong, PR China \\ 3 National Astronomical Observatories, Chinese Academy of Sciences, Beijing 100012, PR China
}

Received 2 June 2005 / Accepted 20 September 2005

\section{ABSTRACT}

\begin{abstract}
Observations of a strong and extended positron-electron annihilation line emission in the Galactic center (GC) region by the Spectrometer on the International Gamma-Ray Astrophysical Laboratory (SPI/INTEGRAL) are challenging to the existing models of positron sources in the Galaxy. In this paper, we study the possibility that pulsar winds in the GC produce the $511 \mathrm{keV}$ line. We propose that three possible scenarios of pulsar winds may exist as the positron sources: normal pulsars, rapidly spinning strongly magnetized neutron stars (magnetars) in gammaray burst (GRB) progenitors, a population of millisecond pulsars in the Galactic center. These $\mathrm{e}^{ \pm}$pairs could be trapped in the region by the magnetic field in the Galactic center, and cool through synchrotron radiation and Coulomb interactions with the medium, thereby becoming non-relativistic particles. The cooling timescales are shorter than the diffuse timescale of positrons, so low-energy positrons could annihilate directly with electrons into $511 \mathrm{keV}$ photons or could form positronium before annihilation. We find that normal pulsars cannot be a significant contributor to the positron sources. Although magnetars in the GC could be potential sources of positrons, their birth rate and birth locations may pose some problems for this scenario. We believe that the most likely candidates for positron sources in the GC may be a population of millisecond pulsars in the GC. Our preliminary estimations predict that the $\mathrm{e}^{ \pm}$annihilation rate in the GC is $\geq 5 \times 10^{42} \mathrm{~s}^{-1}$, which is consistent with the present observational constraints. Therefore, the $\mathrm{e}^{ \pm}$pairs from pulsars winds can contribute significantly to the positron sources in the Galactic center region. Furthermore, since the diffusion length of positrons is short, we predict that the intensity distribution of the annihilation line should follow the distribution of millisecond pulsars, which should then correlate to the mass distribution in the GC.
\end{abstract}

Key words. Galaxy: center - gamma-rays: theory - pulsars: general

\section{Introduction}

Since the first detection (Johnson \& Haymes 1973) and subsequent identification (Leventhal et al. 1978) of the Galactic $511 \mathrm{keV}$ annihilation line, the origin of the galactic positrons has become a lively topic of scientific debate. With the launch of the INTEGRAL gamma-ray observatory in 2002, the SPI board on telescope provides a strong constraints on the morphology and intensity of the $511 \mathrm{keV}$ line emission from the Galactic center (Knödlseder et al. 2003; Jean et al. 2003). The data analyses show that the line emitting source is diffuse, and that the line flux within $5^{\circ}$ of the GC amounts to $\sim(9.9 \pm 4) \times 10^{-4}$ photon $\mathrm{cm}^{-2} \mathrm{~s}^{-1}$ (Knödlseder et al. 2003), corresponding to a luminosity of $\sim 10^{36} \mathrm{erg} \mathrm{s}^{-1}$. The high line luminosity requires that the positron injection rate in the GC should be around (3-6) $\times 10^{42} \mathrm{e}^{+} \mathrm{s}^{-1}$. Recently, analyses of the observational data by SPI/INTEGRAL with deep Galactic center region exposure (longer than $4 \mathrm{Ms}$ ) show that the spatial distribution of $511 \mathrm{keV}$ line appears centered on the Galactic center (bulge component), with no contribution from a disk component (Teegarden et al. 2005; Knödlseder et al. 2005; Churazov et al. 2005). Analysis by Churazov et al. (2005) suggests that the positron injection rate is up to $10^{43} \mathrm{e}^{+} \mathrm{s}^{-1}$ within $\sim 6^{\circ}$, and an intrinsic annihilation line width $\sim 2.37 \pm 0.25 \mathrm{keV}$.

The potential positron sources include: neutron stars or black holes (Lingenfelter \& Ramaty 1983); ${ }^{56}$ Co $\beta$-decays in supernova remnants (Lingenfelter \& Ramaty 1979; Ellison et al. 1990); other radioactive nuclei formed by nucleosynthesis in supernova, nova, red giants, and Wolf-Rayet stars (Ramaty et al. 1979); cosmic ray interactions with the interstellar medium (Kozlovsky et al. 1987); pair production by gamma-ray photons interacting with starlight photons in the interstellar medium (Mastichiadis et al. 1991) and gammaray cascades at the polar caps of pulsars (Harding \& Ramaty 1987; Boulares 1989); electron-positron pairs produced by the pulsar winds (Chi et al. 1996); and probably gamma-ray bursts (GRB, Lingenfelter \& Hueter 1984). However, recent results from SPI/INTEGRAL on the $511 \mathrm{keV}$ annihilation line emission in the Galactic center show a diffuse source with high line luminosity, and present a challenge to the existing 
models of the origin of galactic positrons. As a result, Cassé et al. (2004) argues that normal supernova explosion in the GC cannot contribute to the required positrons, but hypernovae (Type Ic supernovae/gamma-ray bursts) in the Galactic center may be possible sources of galactic positrons. In addition, annihilations of light dark matter particles into $\mathrm{e}^{ \pm}$pairs (Boehm et al. 2004) have also been suggested as the potential origin of the $511 \mathrm{keV}$ line in the GC.

In this paper, we study the possible contribution to positrons in the Galactic center by pulsar winds. We suggest that there are three possibilities of pulsar winds as positron sources in the Galactic center: normal pulsars (e.g. Crab and Vela like pulsars), the rapidly spinning strongly magnetized neutron stars that are possible GRB progenitors (Usov 1992), and a millisecond pulsar population (Wang et al. 2005). In Sect. 2, we study the possibility that electron-positron pairs can be produced by the pulsar winds. In Sect. 3, we discuss in detail how electron-positrons may be produced in three possible scenarios. These $\mathrm{e}^{ \pm}$pairs could be trapped in the Galactic center by the magnetic field. In Sect. 4, we find that both the synchrotron cooling timescale and energy loss timescale by Coulomb interactions in the medium are less than the diffuse timescale of electron-positrons in the GC, so that $\mathrm{e}^{ \pm}$pairs will become nonrelativistic, and then annihilate into $511 \mathrm{keV}$ emission lines. The summary and discussions follow in Sect. 5.

\section{Electron-positron winds from pulsars}

It is well known that relativistic particles from pulsar winds interacting with the interstellar medium form the synchrotron wind nebulae (see reviews by Arons 1998; Kaspi et al. 2004). Chi et al. (1996) proposed that the bulk of the comic positrons could originate from pulsar winds. In this paper, we consider that the electron-positron pair production occurs in the pulsar outer-magnetospheric region, the so-called outer gap (Cheng et al. 1986).

Zhang \& Cheng (1997) have developed a self-consistent mechanism to describe the high energy radiation from spinpowered pulsars. In their model, relativistic charged particles from a thick outer magnetospheric accelerator (outer gap) radiate through the synchro-curvature radiation mechanism (Cheng \& Zhang 1996) rather than through the synchrotron and curvature mechanisms in general, thereby producing non-thermal photons from the primary $\mathrm{e}^{ \pm}$pairs along the curved magnetic field lines in the outer gap. The criterion for the existence of the outer gap requires the fractional size of this outer gap $f<1$, which is the ratio between the mean vertical separation of the outer gap boundaries in the plane of the rotation axis and the magnetic axis to the light cylinder radius. There $f$ is limited by the pair production between the soft thermal X-rays from the neutron star surface and the high energy gamma-ray photons emitted from the outer gap region, and it can be approximated as $f \simeq 5.5 P^{26 / 21} B_{12}^{-4 / 7}$ (Zhang \& Cheng 1997), where $P$ is the spin period, and $B_{12}$ is the surface magnetic field in units of $10^{12} \mathrm{G}$.

The pair production mechanism is a synchrotron photon cascade in a strong magnetic field. According to
Halpern \& Ruderman (1993), each primary electron-positron from an outer gap will have an energy

$E_{\mathrm{p}}=\gamma_{\mathrm{p}} m_{\mathrm{e}} c^{2}=5.7 \times 10^{12} P^{1 / 3} \mathrm{eV}$

before it strikes the neutron star surface, where $\gamma_{\mathrm{p}}$ is the Lorentz factor of the primary electrons, $m_{\mathrm{e}}$ the rest mass of the electron, and $c$ the light speed. These primary electron-positrons will continue to emit curvature photons with a typical energy

$E_{\text {cur }}=\frac{3}{2} \gamma_{\mathrm{p}}^{3} \frac{c}{S} \hbar=6.4 \times 10^{8} P^{1 / 2} \mathrm{eV}$,

where $\hbar$ is the Planck constant, $s=\left(R R_{\mathrm{L}}\right)^{1 / 2}$ the curvature radius, $R=10^{6} \mathrm{~cm}$ the radius of the neutron star, and $R_{\mathrm{L}}=c P / 2 \pi$ is the light cylinder distance. These curvature photons will be converted into secondary $\mathrm{e}^{ \pm}$pairs in the local magnetic field when their energy satisfies (Ruderman \& Sutherland 1975)

$E \geq E_{\text {crit }} \equiv \frac{2 m_{\mathrm{e}} c^{2}}{15} \frac{B_{q}}{B_{s}}=3 B_{s, 9}^{-1} \mathrm{GeV}$,

where $B_{q}=4.4 \times 10^{13} \mathrm{G}$ is a critical magnetic field, and $B_{s, 9}$ is the local surface magnetic field of the neutron star in units of $10^{9} \mathrm{G}$.

It has been proposed that there is a strong multipole magnetic field near the stellar surface, although a global dipole magnetic field gives a good description of the magnetic field far from the star (Ruderman \& Sutherland 1975; Ruderman 1991; Arons 1993). The typical radius of curvature $l$ of the local magnetic field is on the order of the crust thickness of the star (i.e. $l \sim 10^{5} \mathrm{~cm}$ ), which is much less than the dipole radius of curvature $s$ of dipole field component near stellar surface. The relation between the local multipole magnetic field and dipole field can be given by (Zhang \& Cheng 2003)

$B_{s} \simeq B_{\mathrm{d}}\left(\frac{R}{l}\right)^{3}$.

Here $B_{\mathrm{d}}$ is the dipole magnetic field of a pulsar, so the critical energy is rewritten as

$E_{\text {crit }}=\frac{2 m_{\mathrm{e}} c^{2}}{15} \frac{B_{q}}{B_{\mathrm{d}}}\left(\frac{R}{l}\right)^{-3}$

The secondary $\mathrm{e}^{ \pm}$pairs will lose their energy via synchrotron radiation with a photon energy at a distance of $r$

$E_{\text {syn }}=\frac{3}{2}\left(\frac{E_{\text {cur }}}{2 m_{\mathrm{e}} c^{2}}\right)^{2} \frac{e \hbar B(r)}{m_{\mathrm{e}} c}=2.6 \times 10^{5} B_{\mathrm{d}, 9} P\left(\frac{3 R}{r}\right)^{3}\left(\frac{R}{l}\right)^{3} \mathrm{eV}$. (6)

We can see that generally $E_{\text {syn }}>E_{\text {crit }}$, so a photon-electron cascade will start and develop until this condition fails. At the end of a cascade, each incoming primary electron-positron can produce, on average,

$N_{\mathrm{e}^{ \pm}}=\frac{E_{\mathrm{p}}}{E_{\text {crit }}}=1.9 \times 10^{3} B_{\mathrm{d}, 9} P^{1 / 3}\left(\frac{R}{l}\right)^{3}$,

and then the total pair production rate can be estimated as

$\dot{N}_{\mathrm{e}^{ \pm}}=f \dot{N} N_{\mathrm{e}^{ \pm}}=2 \times 10^{33} f B_{\mathrm{d}, 9}^{10 / 7} P^{-8 / 21}\left(\frac{R}{l}\right)^{30 / 7} \mathrm{~s}^{-1}$, 
where

$\dot{N}=2.7 \times 10^{27} P^{-2} B_{\mathrm{d}, 9}\left(\frac{R}{l}\right)^{3} \mathrm{~s}^{-1}$

is the primary electron-positrons passing through the polar gap (Goldreich \& Julian 1969).

Since these pairs are created close to the stellar surface and the field lines are converging, only a small fraction may keep moving toward the star and annihilate on the stellar surface. Ho (1986) showed that the loss cone for these pairs will approach $\pi / 2$ (cf. Eq. (29) of Ho 1986), in other words, most pairs will be reflected by the magnetic mirroring effect and then move toward the light cylinder. These particles will flow out with the pulsar wind and be accelerated by the low-frequency electro-magnetic wave. In theory, the maximum Lorentz factor of pulsar wind particles could be larger than $10^{4}$ (Rees \& Gunn 1974; Kennel \& Coroniti 1984).

\section{Three possible scenarios for pulsar winds in GC}

As discussed in the introduction, we consider three possibilities of positron sources from pulsar winds in the Galactic center region. In this section, we discuss them separately in detail.

\subsection{Normal pulsars}

The deep radio survey (LaRosa et al. 2000) and recent observations in the Galactic center field targeted in the deep Chandra X-ray surveys (Wang et al. 2002a; Muno et al. 2003) show that a few supernova remnants are present. Detailed analyses of X-ray nebulae G0.13-0.11, G359.89-0.08 and G359.54+0.18 (Wang et al. 2002b; Lu et al. 2003) suggested young pulsars might lie inside them. In the past years, young pulsars may have been actively produced in the Galactic center region. However, because the average proper motion velocity of normal pulsars is high $\left(\sim 500 \mathrm{~km} \mathrm{~s}^{-1}\right)$, few of them $(\leq 40 \%)$ lie in the GC (Arzoumanian et al. 2002), so the number of normal pulsars whose active timescale is $\sim 1$ Myr in the GC should be small, and the number of Crab or Vela like pulsars is around 10 (Wang et al. 2005).

There is both theoretical and experimental evidence that young pulsars can emit intense electron/positron winds. In the case of Crab and Vela, the $\mathrm{e}^{ \pm}$injection rates are up to $\sim 10^{41} \mathrm{e}^{+} \mathrm{s}^{-1}$ and $\sim 10^{39} \mathrm{e}^{+} \mathrm{s}^{-1}$. Then in the timescale of the age of Crab or Vela, the total number of positrons is about $3 \times 10^{51}$. Assuming that the birth rate of young pulsars is 1 per $10^{3}$ years in the $\mathrm{GC}$, and that the lifetime of positrons is 1 million years (cf. the cooling time scale estimate in Sect. 4), there will be about $3 \times 10^{54}$ positrons in the region. On average the annihilation rate is $\sim 3 \times 10^{54} / 1$ million $\mathrm{yr} \sim 10^{41} \mathrm{~s}^{-1}$. Therefore, our analysis suggests that the young pulsar winds could not be a significant contributor to the positrons in the Galactic center region.

\subsection{Millisecond magnetar in a GRB progenitor}

It has been suggested that gamma-ray bursts in the GC are potential positron sources (Cassé et al. 2004; Parizot et al. 2005).
Their models assume that the annihilation line may relate to nucleosynthesis in the hypernova that is the GRB progenitor. But the GRB progenitor could also be a rapidly spinning, strongly magnetized neutron star (millisecond magnetar, see Usov 1992; Thompson 1994), that is also a very strong $\mathrm{e}^{ \pm}$pair emitter. The generic magnetized $\mathrm{e}^{ \pm}$outflow from a spinning compact object could be given by

$L_{\mathrm{m}} \simeq 2 \pi r_{0}^{2} c\left(\frac{B^{2}}{4 \pi}\right) \sim 1.5 \times 10^{52} r_{06}^{2} B_{15}^{2}\left(t / t_{0}\right)^{-\alpha} \mathrm{erg} \mathrm{s}^{-1}$

where $r_{0}$ is the size of the central engine, $\sim 10^{6} \mathrm{~cm} ; B \sim$ $10^{15} \mathrm{G}$ is the magnetic field of magnetars; $t_{0}$ is a characteristic timescale after the burst, i.e. $t_{0} \sim 1 \mathrm{~s}$, where we have assumed the magnetic field decay would follow a power law and $\alpha>1$. Assuming the average energy of positrons is $\sim 1 \mathrm{GeV}$, the total number of positrons emitted by magnetars is

$N \sim \int_{t_{0}}^{\infty} 2 \times 10^{55}\left(t / t_{0}\right)^{-\alpha} \mathrm{d} t \sim 2 \times 10^{55} /(\alpha-1)$.

If we take $\alpha=1.1$, the total $\mathrm{e}^{ \pm}$number is up to $2 \times 10^{56}$. For other possible values of $\alpha$, the total $\mathrm{e}^{ \pm}$number will be reduced by a factor of several times. On the other hand, the toroidal magnetic field of magnetars can reach $10^{17} \mathrm{G}$, and part of this toroidal magnetic field energy can also be converted into $\mathrm{e}^{ \pm}$ (Kluzniak \& Ruderman 1998; Dai \& Lu 1998).

Therefore, in this model more than $10^{56}$ positrons would be produced. Because the GRB birth rate from the present observations is $\sim 10^{-5} \mathrm{yr}^{-1}$ in a galaxy (Piran 2004) after considering the beaming effect correction, we expect the GRB rate in the GC is about one per $10^{6}$ years. Therefore, the annihilation rate of positrons produced by the magnetar is $\sim 3 \times$ $10^{42} \mathrm{~s}^{-1}$. But more comments on the GRB rates are required here. Observations of the long GRBs and host galaxies are in agreement with a correlation of GRB locations with blue regions/spirals of host galaxies (Bloom et al. 2002), which implies that long bursts do not concentrate in the bulge region. Theoretical studies of merging neutron stars and black holes suggest that most short bursts may be located in the halo region (e.g. Voss \& Tauris 2003), so here we may have overestimated the GRB rate in the Galactic center region considering the uncertainties of the burst sites of different GRB populations.

\subsection{A millisecond pulsar population}

We have argued that millisecond pulsars could be the major contributor to the pulsar population in the Galactic center (see Wang et al. 2005). There may exist a population of old neutron stars with low space velocities that have not escaped the Galactic center (see Belczynski \& Taam 2004a,b). Such neutron stars could be recycled to millisecond periods, and could remain in the GC through their lifetime because of their relatively low proper motion velocity (Lyne et al. 1998; Arzoumanian et al. 2002). Wang et al. (2005) suggest that the millisecond pulsar population can contribute significantly to the diffuse gamma-rays in the Galactic center (Mayer-Hasselwander et al. 1998). In addition, the X-ray synchrotron nebula around the millisecond pulsar B1957+20 
(Stappers et al. 2003) provides evidence that millisecond pulsars can remain active enough to emit relativistic winds. Therefore, we propose that electron-positrons from the winds of the millisecond pulsar population could be the possible positron sources in the GC.

Since millisecond pulsars can lie in the GC and remain active for $1 \mathrm{Gyr}$, we believe they are continuous positron injecting sources. For the typical parameters of millisecond pulsars, $P=3 \mathrm{~ms}, B_{\mathrm{d}} \sim 3 \times 10^{8} \mathrm{G}$ and according to Eq. (8), we estimate the positron injection rate $\dot{N}_{\mathrm{e}^{ \pm}} \sim 5 \times 10^{37} \mathrm{~s}^{-1}$ for a millisecond pulsar. Wang et al. (2005) showed that about 6000 millisecond pulsars could contribute to the diffuse gamma-rays in the Galactic center region covered by EGRET. Since the present annihilation observations show that the line emission region toward GC is about $6^{\circ}$, which is much larger than the EGRET observation region $\left(1.5^{\circ}\right)$, a larger number of MSPs are expected to lie in the bulge region of $6^{\circ}$. We do not know the distribution of MSPs in GC, so we just scale the number of MSPs by $6000 \times\left(6^{\circ} / 1.5^{\circ}\right)^{2} \sim 10^{5}$, where we assume the number density of MSPs may be distributed as $\rho_{\text {MSP }} \propto r_{\mathrm{c}}^{-1}$, where $r_{\mathrm{c}}$ is the scaling size of GC. Based on the population analysis of Lyne et al. (1998), the number of millisecond pulsars in the entire Galaxy may exceed $3 \times 10^{5}$, so the number of millisecond pulsars in the bulge region estimated here could be reasonable. The total positron injection rate from the millisecond pulsar population is thus $\sim 5 \times 10^{42} \mathrm{e}^{+} \mathrm{s}^{-1}$.

\section{Electron-positron cooling in GC and annihilations}

The outflowing electron-positrons could be trapped by the magnetic field in the Galactic center. The Larmor radius $\left(r_{\mathrm{L}}\right)$ of a relativistic electron with energy $E_{\mathrm{e}}$ is given by

$r_{\mathrm{L}} \approx E_{\mathrm{e}} / e B \sim 10^{13}\left(E_{\mathrm{e}} / 10^{10} \mathrm{eV}\right)\left(B / 10^{-5} \mathrm{G}\right)^{-1} \mathrm{~cm}$.

The angular size of $\mathrm{e}^{ \pm}$annihilation line emission detected by SPI/INTEGRAL is several degrees (Knödlseder et al. 2003), so we take $\lambda \sim 300 \mathrm{pc}$ as the size of the Galactic center region studied in this paper (assuming the distance of the GC to us $\sim 8 \mathrm{kpc}$ ). The diffuse timescale of the electron is estimated as

$t_{\text {diff }} \sim\left(\frac{\lambda}{r_{\mathrm{L}}}\right)^{2} \frac{r_{\mathrm{L}}}{c} \sim 10^{10}\left(E_{\mathrm{e}} / 10^{10} \mathrm{eV}\right)^{-1} \mathrm{yr}$,

where we take the average magnetic field in the GC $B \sim 10^{-5} \mathrm{G}$ (Uchida \& Güsten 1995; LaRosa et al. 2005) and $\lambda \sim 10^{21} \mathrm{~cm}$.

Electrons and positrons in the Galactic center will lose their energy via synchrotron radiation and Coulomb interactions with the medium, and then annihilate into $511 \mathrm{keV}$ photons. If we assume the injection electron-positron energy spectrum in the form $\dot{Q}_{\mathrm{e}^{ \pm}} \sim A E_{\mathrm{e}}^{-p}$, where $p \geq 2$, then positrons with energies higher than $\sim 100 \mathrm{MeV}$ will cool through synchrotron radiation in the magnetic field, while a large number of low energy positrons lower than $\sim 100 \mathrm{MeV}$ lose their energy by Coulomb interactions in the medium of the GC. The other energy-loss processes, like inverse Compton cooling and bremsstrahlung, are negligible. When the positrons reach an energy around a few tens of eV, they could either annihilate directly with electrons or form positronium by charge exchange or radiative capture (Bussard et al. 1979). Thus the annihilation timescales depend on the cooling timescales.

The synchrotron cooling timescale of relativistic positrons is estimated as

$\tau_{\mathrm{s}} \simeq \frac{\gamma m_{\mathrm{e}} c^{2}}{\gamma^{2}\left(\frac{e B}{m_{\mathrm{e}} c}\right)^{2} \frac{e^{2}}{c}} \sim 3 \times 10^{6}\left(E_{\mathrm{e}} / 10^{10} \mathrm{eV}\right)^{-1} B_{-5}^{-3 / 2} \mathrm{yr}$.

We find that the electron synchrotron timescale is lower than the diffusion timescale for the same electron energy, $\tau_{\mathrm{s}} \ll t_{\text {diff }}$, so the positrons could significantly lose their energy to become non-relativistic and stay in the Galactic center.

The energy loss timescale of Coulomb interactions in the medium $\tau_{\mathrm{c}}$ depends on the positron energy, the medium density, and the degree of ionization. For the positrons with energy around $1-100 \mathrm{MeV}$, the Coulomb interaction cooling timescale is estimated as $\tau_{\mathrm{c}} \sim 10^{5} n^{-1} \mathrm{yr}$ (within a factor of 2 ), and $n$ is the medium number density in units of $\mathrm{cm}^{-3}$. However, the medium in GC is quite complicated, as many giant molecular clouds (LaRosa et al. 2000) exist, as does hot gas discovered in X-rays (Muno et al. 2004). Then the energy loss timescales could vary from $\tau_{\mathrm{c}}<10^{3} \mathrm{yr}$ in the molecular clouds $(n>$ $\left.10^{2} \mathrm{~cm}^{-2}\right), \tau_{\mathrm{c}} \sim 10^{5} \mathrm{yr}$ for the typical warm interstellar medium $\left(n \sim 1 \mathrm{~cm}^{-2}\right)$, and $\tau_{\mathrm{c}}>10^{7} \mathrm{yr}$ for the hot gas $\left(n \sim 10^{-2} \mathrm{~cm}^{-2}\right)$. Analysis of the $511 \mathrm{keV}$ annihilation spectrum suggests that the dominant fraction of positrons ( $94 \%)$ form positronium before annihilation, which constrains the positron annihilation in the hot gas to a very small fraction $(<8 \%$, see Churazov et al. 2005). Most positron annihilations seem to lie in the warm medium, so the cooling timescale is also much shorter than the diffusion timescale.

\section{Summary and discussions}

The origin of Galactic positrons is still a mystery at present. In this paper, we have proposed a possible contribution to positron sources by the pulsar winds in the region of the Galactic center. We discussed the contributions of three possible pulsar scenarios in the GC: normal pulsars, the rapidly spinning strongly magnetized neutron stars in GRB progenitors (millisecond magnetars), and a millisecond pulsar population. Electron-positron pairs are injected into the Galactic center region from three classes of pulsar winds, and could be trapped in this region by the magnetic field in the Galactic center. These relativistic $\mathrm{e}^{ \pm}$pairs will lose their energy though the synchrotron radiation and the Coulomb interactions with the medium to become non-relativistic. The cooling timescales are shorter than the diffusion timescale of positrons in the magnetic field in the GC, so positrons could form positronium by charge exchange or radiative capture, or annihilate directly with electrons into photons to produce the $511 \mathrm{keV}$ line observed by the present detectors. Our results have shown that normal pulsars cannot be a significant contributor to positron sources in the GC, but the millisecond magnetar and a millisecond pulsar population could be potential positron sources. However, as we estimate that the diffusion time is much longer than the cooling time, it is not clear how the positrons produced by GRBs 
can spread over the entire galactic bulge or at least do so in the range of $6^{\circ}$. Parizot et al. (2005) argue that the turbulent diffusion process is able to diffuse the positrons over the galactic bulge in a timescale of $10^{7} \mathrm{yr}$. However, it is still not clear why the positrons produced by GRBs should concentrate in the galactic bulge, and this is not supported by the observations of GRBs and their host galaxies (e.g. long bursts in Bloom et al. 2002; short bursts in Gehrels et al. 2005). In view of the uncertainty of the birth rate of GRBs in the GC and annihilation lines lacking in the disk, we must have reservations about GRBs as the significant positron sources in the GC.

The predicted annihilation rate of positrons from the millisecond pulsar population is $\sim 5 \times 10^{42} \mathrm{~s}^{-1}$ which is consistent with observational constraints by SPI/INTEGRAL. We therefore conclude that the $\mathrm{e}^{ \pm}$pairs from these classes of pulsar winds are potential positron sources that can significantly contribute to the $511 \mathrm{keV}$ annihilation lines in GC.

The important problem for positrons is determineing the $\mathrm{e}^{+} \mathrm{e}^{-}$annihilation line intensity and radiation morphology in the Galaxy. Recently, analyses of the data by SPI/INTEGRAL with deep Galactic center region exposure show that the spatial distribution of $511 \mathrm{keV}$ line appears centered on the Galactic center (bulge component), with no contribution from a disk component (Teegarden et al. 2005; Knödlseder et al. 2005; Churazov et al. 2005). We suggested that the positrons produced by pulsar winds could be trapped in the Galactic center by the magnetic field, and the diffusion timescale is higher than the cooling timescales, so that the positrons can annihilate into $511 \mathrm{keV}$ photons before they can escape from the Galactic center region. In the other viewpoints, the bulgedominated morphology of the $511 \mathrm{keV}$ line may indicate that the positron source population could be an old stellar population. Knödlseder et al. (2005) suggest that the low-mass $\mathrm{X}$-ray binaries (LMXBs) could be the candidate sources because more than $60 \%$ of the Galactic LMXBs are observed towards the galactic bulge (see Grimm et al. 2002). The millisecond pulsar population is a very old stellar population, and part of them stay in LMXBs, but most may be isolated as radio pulsars, X-ray sources, and be even detectable in gamma-rays. Recent radio observations find most millisecond pulsars lie in globular clusters (see the review by Camilo \& Rasio 2005, and references therein), with few of them in the Galactic field. If assuming the millisecond pulsar evolutionary formation channels in globular clusters are similar to the Galactic center region or bulge region, then we expect the millisecond pulsar population in the GC as a significant contributor to the positron sources. The millisecond pulsars can stay in the Galactic center region throughout their lifetime, which is well consistent with the 511 line emission morphology.

We have shown that the millisecond pulsar population in the Galactic center could provide the major sources of positrons. Normal pulsars could not be the main positron sources, but GRB progenitors,i.e. millisecond magnetars, may be significant contributors. In addition, hypernovae/GRBs could also be the potential positron sources (Cassé et al. 2004). Thus, how could we distinguish the model of a millisecond pulsar population from other models, especially models related to GRBs? Firstly, we can estimate the typical spatial diffusion scale, according to Eq. (13), $\lambda_{\text {diff }} \sim\left(r_{\mathrm{L}} c t\right)^{1 / 2}$. The average cooling time of positrons in the GC is $10^{6}$ years (Churazov et al. 2005), so the characteristic diffusion scale is about $10^{18} \mathrm{~cm}$. Because of the low angular resolution of SPI/INTEGRAL (about 2 degrees, Vedrenne et al. 2003), we can assume that the positrons annihilate in the same local region as their sources, i.e. the millisecond pulsars. Therefore we predict that the spatial intensity distribution of the annihilation lines should follow the spatial distribution of MSPs if a millisecond population exists in the GC. Additionally, we could assume the spatial distribution of MSPs should follow the mass distribution of the GC though we do not know how well they follow each other. But because the proper motion velocity of MSPs is relatively low, we could reasonably assume that the two distributions are quite close to each other. Therefore, if the positron sources originate in the MSP population, the $511 \mathrm{keV}$ annihilation line intensity would follow the mass distribution of the Galactic center region. The present image of $511 \mathrm{keV}$ line emission (see Figs. 4 and 6 in Knödlseder et al. 2005) shows that the flux is strongest in the center and decreases toward the outside region, possibly tracing the mass distribution of the GC, but this needs confirmation by future high resolution observations. The millisecond pulsar population could naturally explain the diffuse emission morphology and does not have the problem of the turbulent diffusion, which is required to diffuse all these positrons to a few hundred pc. On the other hand, for models associated with GRBs, the positron source is initially a point-like source, so the diffuse emission of $511 \mathrm{keV}$ line needs the strong turbulent diffusion to diffuse the positrons on to a larger scale (Parizot et al. 2005). Thus far, GRB progenitors (either magnetars or hypernovae) are related to massive stars and dense clouds (e.g. molecular clouds in the GC that has a dimension of a few tens pc, LaRosa et al. 2000), the mean density $n>10^{2} \mathrm{~cm}^{-3}$, and the cooling time of positrons is less than $10^{3}$ years. Thus the spatial diffusion scale is only $10^{17} \mathrm{~cm}$, which makes the annihilation line emission look like a point source that is correlated with the molecular clouds. In order to make the line emission be a diffuse source, GRB positrons must diffuse to a hundred pc with a long diffusion timescale $>10^{7}$ years, so it requires that the mean density in the $\mathrm{GC}$ is $\sim 10^{-2} \mathrm{~cm}^{-3}$. But this low density seems unreasonable in the $\mathrm{GC}$, and it is also not consistent with the constraint on the medium environment from the spectrum of $511 \mathrm{keV}$ line (Churazov et al. 2005). Furthermore, if positrons cannot diffuse to larger distances, the intensity distribution of annihilation lines should not correlate with the general mass distribution of the GC; instead, it should be more correlated to the distribution of massive stars and dense molecular clouds. Moreover, GRBs occur more likely in the disk, where more dense molecular clouds and massive stars are located, and in fact, the detected magnetars and soft gamma-ray repeaters are also distributed in the disk. Giant flares from soft gamma-ray repeaters (SGR) are also positron source candidates that are distributed in the Galactic plane, but the $511 \mathrm{keV}$ line observations show a very weak disk component (Teegarden et al. 2005; Knödlseder et al. 2005). The 27 Dec. giant flare from SGR 1806-20 released an energy up to $10^{47}$ erg (Hurley et al. 2005), and it is thought to be a possible origin of short GRBs, so might be considered 
as the $511 \mathrm{keV}$ emission source in the future. In summary, the positron source models associated with GRBs are disputable. Our scenario of a millisecond pulsar population as possible positron sources in the GC has some advantages of explaining the diffuse morphology of $511 \mathrm{keV}$ line emissions, and it predicts that the line intensity distribution should follow the mass distribution of the GC, which may be tested by future high resolution observations.

Acknowledgements. We are grateful to the referee for the critical comments, to R. Diehl, Y. F. Huang and A. Strong for the discussions. This work was supported by an RGC grant of the Hong Kong Government under HKU 7015/05P and the National Natural Science Foundation of China under grant 10273011 and 10573021.

\section{References}

Arons, J. 1998, preprint [arXiv: astro-ph/9809392]

Arzoumanian, Z., Chernoff, D. F., \& Cordes, J. M. 2002, ApJ, 564, 333

Belczynski, K., \& Taam, R. E. 2004a, ApJ, 603, 690

Belczynski, K., \& Taam, R. E. 2004b, ApJ, submitted [arXiv: astro-ph/0311287]

Bloom, J. S., Kulkarni, S. R., \& Djorgovski, S. G. 2002, AJ, 123, 1111

Boehm, C., et al. 2004, Phys. Rev. Lett., 92, 101301

Boulares, A. 1989, ApJ, 342, 807

Bussard, R. W., Ramaty, R., \& Drachman, R. J. 1979, ApJ, 471, 673

Camilo, F., \& Rasio, F. A. 2005, preprint [arXiv: astro-ph/0501226]

Cassé, M., Cordier, B., Paul, J., \& Schanne, S. 2004, ApJ, 602, L17

Cheng, K. S., Ho, C., \& Ruderman, M. A. 1986, ApJ, 300, 500

Cheng, K. S., et al. 1990, J. Phys. G: Nucl. Part. Phys., 16, 1115

Cheng, K. S., \& Zhang, L. 1996, ApJ, 463, 271

Chevalier, R. A. 2000, ApJ, 539, L45

Chi, X., Cheng, K. S., \& Young, E. C. M. 1996, ApJ, 459, L83

Churazov, E., Sunyaev, R., Sazonov, S., Revnivtsev, M., \& Varshalovich, D. 2005, MNRAS, 357, 1377

Dai, Z. G., \& Lu, T. 1998, Phys. Rev. Lett., 81, 4301

Ellison, D. C., Frank, J. C., \& Ramaty, R. 1990, in Proc. 21st Int. Cosmic-Ray Conf., ed. R. J. Protheroe (Adelaide: Univ. Adelaide), 4

Gehrels, N., et al. 2005 [arXiv: astro-ph/0505630]

Goldreich, P., \& Julian, W. H. 1969, ApJ, 157, 859

Grimm, H. J., Gilfanov, M., \& Sunyaev, R. 2002, A\&A, 391, 923

Halpern, J. P., \& Ruderman, M. 1993, ApJ, 415, 286

Harding, A. K., \& Ramaty, R. 1987, in Proc. 20th Int. Cosmic-Ray Conf. (Moscow: Nauka), 2

Ho, C. 1986, MNRAS, 221, 523

Hurley, K., Boggs, S. E., Smith, D. M., et al. 2005, Nature, 434, 1098
Jean, K., Knödlseder, J., Lonjou, V., et al. 2003, A\&A, 407, L55

Johnson, W. N., \& Haymes, R. C. 1973, ApJ, 184, 103

Kaspi, V. M., Roberts, M. S. E., \& Harding, A. K. 2004, preprint [arXiv: astro-ph/0402136]

Kennel, C. F., \& Coroniti, F. V. 1984, ApJ, 283, 694

Kluzniak, W., \& Ruderman, M. 1998, ApJ, 508, L113

Knödlseder, J., Lonjou, V., Jean, P., et al. 2003, A\&A, 411, L457

Knödlseder, J., Jean, P., Lonjou, V., et al. 2005, A\&A, 441, 513

Kozlovsky, B., Lingenfelter, R. E., \& Ramaty, R. 1987, ApJ, 316, 801

LaRosa, T. N., Kassim, N. E., Lazio, T. J. W., \& Hyman, S. D. 2000, AJ, 119, 207

LaRosa, T. N., et al. 2005, preprint [arXiv: astro-ph/0505244]

Leventhal, M., MacCallum, C. J., \& Stang, P. D. 1978, ApJ, 225, L11

Lingenfelter, R. E., \& Ramaty, R. 1979, in Proc. 16th Int. Cosmic-Ray Conf., ed. S. Miyake (Tokyo: Tokyo Univ. Press), 1

Lingenfelter, R. E., \& Ramaty, R. 1983, in Positron-Electron Pairs in Astrophysics, ed. M. L. Burn, A. K. Harding, \& R. Ramaty, AIP Conf. Proc., 267

Lingenfelter, R. E., \& Hueter, G. J. 1984, in High-Energy Transients in Astrophysics, ed. S. E. Woosley, AIP Conf. Proc., 558

Lu, F., Wang, Q. D., \& Lang, C. 2003, AJ, 126, 319

Lyne, A. G., Manchester, R. N., Lorimer, D. R., et al. 1998, MNRAS, 295, 743

Mastichiadis, A., Protheroe, R. J., \& Stephen, S. A. 1991, in Proc. 22d Int. Cosmic-Ray Conf., ed. D. O'Sullivan (Dublin: Dublin Institute), 2

Mayer-Hasselwander, H. A., et al. 1998, A\&A, 335, 161

Muno, M. P., Baganoff, F. K., Bautz, M. W., et al. 2003, ApJ, 589, 225

Muno, M. P., Baganoff, F. K., Bautz, M. W., et al. 2004, ApJ, 613, 326

Parizot, E., Cassé, M., Lehoucq, R., \& Paul, J. 2005, A\&A, 432, 889

Piran, T. 2004, Rev. Mod. Phys., 76, 1143

Ramaty, R., Kozlovsky, B., \& Lingenfelter, R. E. 1979, ApJS, 40, 487

Rees, M. J., \& Gunn, J. E. 1974, MNRAS, 167, 1

Ruderman, M. A., \& Sutherland, P. 1975, ApJ, 196, 57

Ruderman, M. A. 1991, ApJ, 366, 261

Stappers, B. W., Gaensler, B. M., Kaspi, V. M., van der Klis, M., \& Lewin, W. H. G. 2003, Science, 299, 1372

Teegarden, B. J., Watanabe, K., Jean, P., et al. 2005, ApJ, 621, 296

Thompson, C. 1994, Mon. N. Royal Astron. Soc., 270, 480

Uchida, K. I., \& Güsten 1995, A\&A, 298, 473

Usov, V. V. 1992, Nature, 357, 472

Vedrenne, G., Roques, J.-P., Schönfelder, V., et al. 2003, A\&A, 411, L63

Voss, R., \& Tauris, T. M. 2003, MNRAS, 342, 1169

Wang, Q. D., Gotthelf, E. V., \& Lang, C. 2002a, Nature, 415, 148

Wang, Q. D., Lu, F., \& Lang, C. 2002b, ApJ, 581, 1148

Wang, W., Jiang, Z. J., \& Cheng, K. S. 2005, MNRAS, 358, 263

Zhang, L., \& Cheng, K. S. 1997, ApJ, 487, 370

Zhang, L., \& Cheng, K. S. 2003, A\&A, 398, 639 